\title{
SCTCMG 2018
}

\section{International Scientific Conference «Social and Cultural Transformations in the Context of Modern Globalism»}

\section{INCREASING COMPETITIVENESS OF THE RUSSIAN SPACE INDUSTRY ENTERPRISE BUSINESS PROCESSES}

\author{
A.V. Kukartsev (a, b), A.A. Boyko (a, b), V.V. Kukartsev (a,b), V. S. Tynchenko (a, b)* and \\ T.V. Dubrovskaya (a) \\ *Corresponding author
}

(a) Reshetnev Siberian State Aerospace University, 31, Krasnoyarsky Rabochy Av., Krasnoyarsk, Russian Federation, 660037

(b) Siberian federal university 79 Svobodny pr., Krasnoyarsk, Russian Federation, 660041

\begin{abstract}
The rocket and space industry is the leading science-intensive branch of the national economy. The enterprises scale and high technology production conduce to high requirements for the management of the rocket and space industry enterprises. Management of innovative business processes of such enterprises requires modern managers to have a high level of modern technologies proficiency and management tools. The Russian space industry enterprises have entered the world market of science-intensive products, and therefore the importance of effective management methods in a market economy not only determines the competitiveness of the organization, but also often solves the problem of domestic enterprises survival in conditions of the world competition. In the article, a brief comparative analysis of scientific approaches and methods of managing innovative business processes in the rocket and space industry enterprises, was conducted. The branches' problems of the defence industry of Russia were examined. The qualities and scope of the "full" and "through" management methods and improvement of the enterprise's innovative business processes were presented. The necessity of enterprise management approach transition from functional to process was substantiated. The algorithm for increasing business competitiveness in the rocket and space industry enterprise was analysed. The stages of regulating the business processes at the manufacturing enterprise by reengineering the Russian space industry enterprise business processes were presented. The article emphasizes the necessity to develop an algorithm for the management of the Russian space industry enterprise's innovative business processes, designed to increase their competitiveness.
\end{abstract}

Keywords: Managing algorithm, innovative business processes, Russian space industry, enterprise business processes, enterprise competitiveness. 


\section{Introduction}

The rocket and space industry is the leading science-intensive branch of the national economy (Baklanov, 2007; Boyko, Kukartsev, Tynchenko, Nasyrov, \& Kukartsev, 2018). Management of innovative business processes of such enterprises requires modern managers to have a high level of modern technologies proficiency and management tools (Chubai, 2010).

The ubiquitous functional approach to management, based on the old management paradigm, has ceased to correspond to the new economic conditions in last decades (Pabian, \& Pabian, 2014, Meskon, Albert, \& Hedouri, 2009). As a result, there were shortcomings in the functional management of the organization: lack of customer orientation, weak delegation of authority and responsibility, bureaucracy, too complex organizational structure, lack of responsibility for the final product (Repin, \& Eliferov, 2013,). The Russian space industry (RSI) enterprises have entered the world market of science-intensive products, and therefore the importance of effective management methods in a market economy not only determines the competitiveness of the organization, but also often solves the problem of domestic RSI enterprises survival in conditions of the world competition (Tarasenko, 1996; Moller, 2006).

\section{Problem Statement}

Speaking of the necessity to develop a methodology for managing innovative business processes of the RSI enterprises, it is necessary to consider an algorithm for increasing its competitiveness based on the use of advanced methods and tools for managing business processes of the enterprise.

Among the invited specialists in the field of the process approach to management, a working group is formed, headed by the project manager (Yeremeev, 2013).

The structure includes subgroups of analysts, performing the main work on business processes. For an average project, the number of analysts, as a rule, is five to seven people. The project curator gives directions to the working group's work, coordinating it with the enterprise manager, the project manager and the heads of the functional divisions of the enterprise (owners of business processes). At the departmental level, analysts work with employees of functional units.

In addition to create a working group for the project, it is necessary to inform the organization's team about the upcoming changes. The manager always encounters the changes' resistance to the project and must effectively overcome it. To do this, one must convincingly explain the directions of forthcoming work, and the employees should believe that organizational changes will not negatively affect their situation. It is necessary to create a favourable psychological climate, encouraging the creative activity of employees, to prepare the ground for less painful organizational changes (Batkovskiy, Makarov, Semenova, Fomina, \& Khrustalev, 2015).

\section{Research Questions}

To analyse the external and internal organization's state, various methods are used: SWOT analysis, PEST environment analysis, Boston matrix, etc., which determine its strengths and weaknesses, opportunities and influences exerted by the environment. At this stage: 
- problems facing the organization, both in its current activities and in the strategic direction, are identified;

- project objectives are determined and ranked;

- tasks are set to improve the functioning of the organization, ways to address them are identified;

- $\quad$ staff is informed about the tasks assigned;

- detailed planning of the work package is carried out.

\section{Purpose of the Study}

At the information stage, extensive databases of business processes are created, which fully describe the work of a science-intensive enterprise. Undoubtedly, this step requires a lot of time and is carried out in several stages (Figure 01).

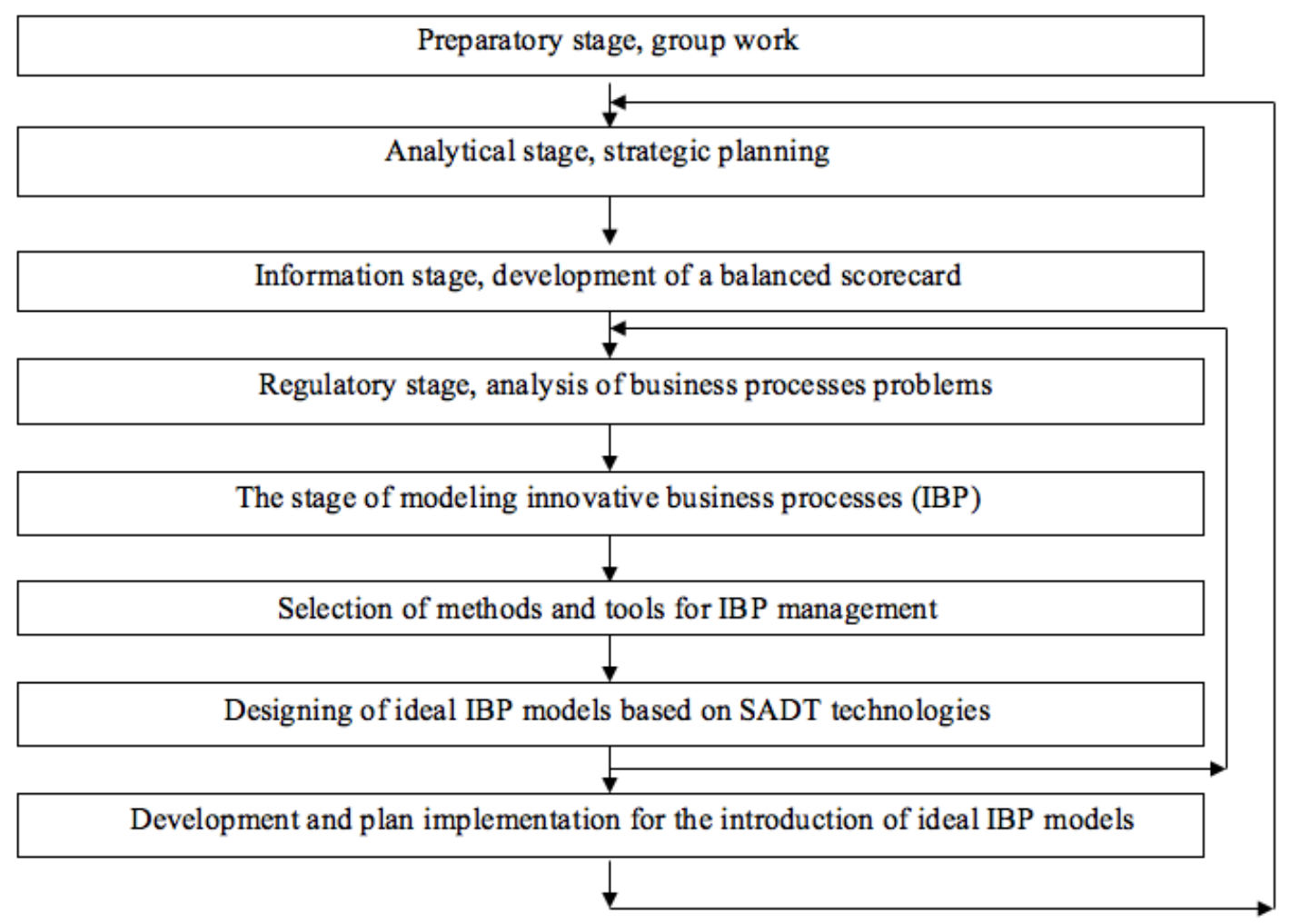

Figure 01. Methodology of the RSI enterprise business process management

\section{Research Methods}

To describe a real model, the actions of enterprise specific employees, as well as their solutions, must be presented. For each step, it is necessary to closely monitor the logical level of the description, i.e. each time, one must clearly visualize the upper level of regulation, or already used business processes of the second and/or third level. It is necessary to describe those operations, actions and works that are not just formalized, but are actually implemented.

Any business process must be analysed in accordance with specified requirements (Gorlevskiya \& Ogurchenok, 2014). These requirements can be established both by the enterprise itself and by external 
organizations. In addition, each business process can be compared with similar processes in other enterprises. In this case, the method of benchmarking business processes can be applied, which consists of the introduction of the best templates of processes samples which are effectively implemented by competitors.

The need for successful operation in a highly competitive environment makes demands on the effectiveness of the RSI enterprises innovative business processes managing (Danilina \& Mingaleva, 2013). The solution of enhancing management efficiency task is closely linked with the provision of information support for business processes (Becker, Vilkova, Taratukhina, Kugeler, \& Rosemann, 2007). One of the main tools for solving this problem is the regulation of enterprise innovative business processes.

There are special types of activities in the RSI enterprises. It is performed in a similar sequence in each structural unit. An example of such business processes is the work with the documentation.

\section{Findings}

The structure of a typical documented procedure has the following form: the purpose of the document and the scope of its application; normative references; special terms and definitions; general description of the documentation procedure; business process responsibility matrix; documenting and archiving; the procedure for amending regulations; distribution of regulations; list of changes; acquaintance of employees; forms of documents.

The rules for the implementation of the business process is a normative and methodical document intended for a comprehensive description of business processes at the top and middle levels. The template for the regulation of the business process should be used to formalize the key business processes of the enterprise. It can be used to regulate both cross-functional (end-to-end) business processes and for processes that are performed within the departments of the RSI enterprise.

To fill the business process regulation template, employees have to search, analyse and systematize information about all main elements of the business process: input (resource), output (product), mechanism (executors and enterprise infrastructure), management (prescriptive documentation). Further, it is necessary to carry out an analysis and to mark the actions of managers and executors in the business process responsibility matrix.

Previous experience of using regulation templates of innovative business processes at the RSI enterprises shows that the labour intensity of filling them is quite high. Each document is difficultly coordinated with all interested employees. The regulation template shows the activity of the business process in the context of the process approach to management. With its help, it is necessary to describe the main business processes of the RSI enterprise.

The stages of the business processes regulation at the RSI enterprise are:

1. Development of the enterprise's regulatory and methodological documentation structure.

2. Development of business processes regulations forms.

3. Development and coordination of procedures for enterprise documentation management.

4. Development and approval of the business processes regulation plan.

5. Development of regulatory and methodological documentation of the company's top level.

6. Development of regulations for the business processes implementation. 
Fig. 2 shows the algorithm for the innovative business process regulation of the RSI enterprise, which can be conditionally called as the development of regulations from scratch.

After collecting the primary information, it is necessary to define and describe the modelled business processes, determine their composition and boundaries. To accomplish this, the external environment of the organization, inputs and outputs and the organizational divisions are identified. The organization is presented in the "black box" form, the functions of each individual division are defined, within which "through" business processes are put in place, the primary liability matrixes of the units are built, and then a composite liability matrix is created. The high complexity of the project and its duration minimize the risk of project implementation to a minimum and ensure sufficient correctness of the process models, and also allows the use of the information obtained in the form of models in the future work.

To define and describe the business processes of an enterprise, it is necessary to determine the characteristics of the production process: to learn the technological process of production or services, the organizational structure, and also the structure of production management. Typically, a description and analysis of a single business process requires about two working days of a highly qualified analyst. Based on the collected data, the initial data are formed for further transformations in business processes.

For a correct and accurate description of the business processes system, a correct and consistent choice of the decomposition strategy is necessary. The most commonly used strategies are: the functional decomposition strategy; the decomposition strategy, based on the analysis of the system life cycle; decomposition based on already known stable subsystems; the P3 decomposition strategy; decomposition according to the physical principle.

Further, the performance indicators of the described business processes are established. The main indicators are the following:

- quantity of products of the required quality;

- the number of consumers;

- cost of production;

- the number of typical operations, necessary for execution in the production for a certain period of time; 


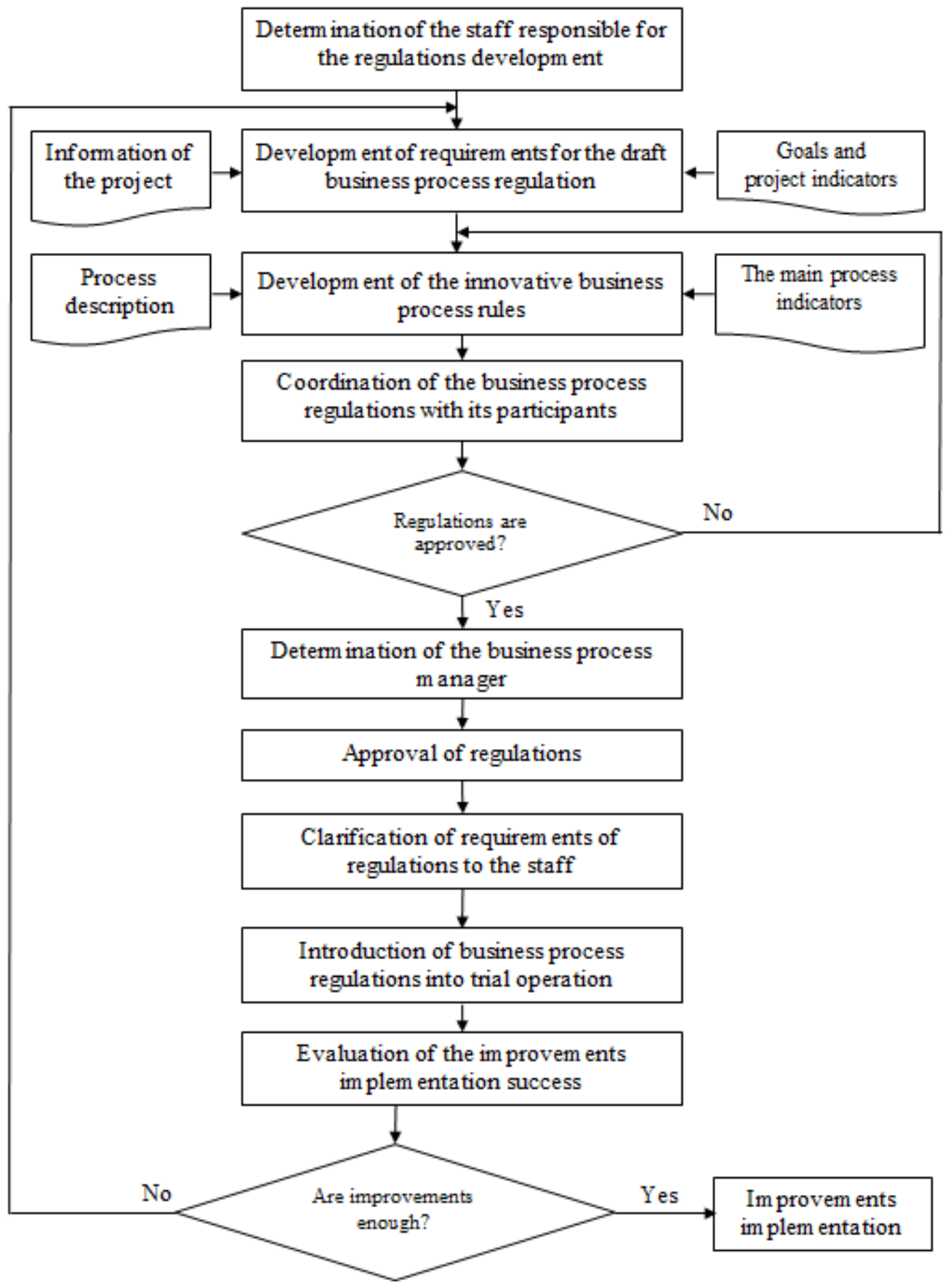

Figure 02. Algorithm for regulation innovative business process

- duration of typical operations;

- productivity of the enterprise personnel;

- $\quad$ investment in production;

- the quality of products generated by the business process, measured using a number of key indicators.

Based on the collected information, quantitative values and a qualitative assessment of these business process efficiency indicators are determined. 
Further, using the SADT methodology, based on the IDEF0 and IDEF3 standards, the working group creates a structurally functional model of real business processes, which, in turn, objectively reflects the totality of the main and auxiliary business processes of the examined object. If necessary, a simulation model can be used to monitor the dynamic changes in the business process system, in addition to the structural-functional model.

The developed business process models must pass the following types of tests: a check on compliance with the standard; verification of adequacy ("author-reader" cycle); verification of correctness (compliance with the Glossary, the basic rules of the standard, etc.); checking for compliance with the requirements of the business process management system (availability of resources, process indicators, management availability, inappropriate products, etc.)

No matter how complex and complete the model is, it is only the image of the real object, and it reflects itself in the presence of certain assumptions. However, until the adequacy of the model is proved to be the real process, it cannot be asserted that with its help the results that really characterize the object under investigation will be obtained. The most important task of modelling business processes is to evaluate the model for adequacy, since every research with an inadequate model does not make sense.

After a comprehensive analysis of the selected business processes problems, it is necessary to choose a method for their improvement. For this, basically two basic methods are possible: business processes reengineering or their continuous improvement.

There are also four main methods for improving innovative enterprise business processes:

- FAST (method of functions system analysis).

- Benchmarking (method of copying a business process).

- BPI (business processes improvement, based on international standards ISO 9000).

- $\quad$ BPR (business processes reorganization).

The foundation of BPI is the gradual and continuous improvement of managed processes. In contrast, BPR is a radical one-time change in the business process by completely restructuring it.

The greatest effect of these techniques is in their integrated use. Initially, the process is improved evolutionarily, then, when there is a gap between the existing process and modern achievements, the process is to be one-time cardinally restructured; after that, the improvement is renewed and the whole cycle is started anew. According to Hammer, the process of this procedure in time is non-uniform: as the various factors and business conditions change, both the processes and their parts can be reconstructed, and the height of the "jump" and the frequency of the process update change.

By methods of BPR or BPI, the business processes of the organization are improved. When improving existing business processes, vertical and horizontal "compression" are used. With vertical "compression", there is a reduction in the levels of the functional hierarchy involved in the business process execution. With horizontal "compression", the execution time and number of functions are reduced, and their efficiency is increased. 
As a result, it should be noted that these two interrelated methods are intended to solve one task which is the development of a science-intensive enterprise based on the improvement of innovative business processes. Therefore, both methods should be used.

Further, depending on the chosen method, based on the analysis of existing business process management tools, the most appropriate tool should be selected. At the strategic level of management, the value chain of business processes is used (M. Porter's methodology), the Work Flow methodology, and the methodology for regulating business processes.

The tools for tactical management of business processes are: a description of the enterprise's activities in the business processes system form, the use of the matrix for the separation of administrative management tasks (DAMT (distribution of administrative management tasks) methodology), as well as a system of business process indicators in conjunction with the KPI methodology (key performance indicators).

For operational management, it is possible to use structural-functional and simulation modelling, as well as CALS-technologies as a means of formalizing all types of activities, automating the management of databases of business processes of the RCI enterprise.

Based on the SADT methodology, based on the IDEF0 and IDEF3 standards, the working group creates a structured and functional model "as it should be", which objectively reflects the totality of the main and auxiliary business processes of the examined object. If necessary, in addition to the structuralfunctional model, to "lose" business processes and monitor the dynamic changes in the aggregate business processes, an imitation model "as is" is created. To build models, All Fusion Process Modeller and Arena are used, by which the Journal of business process models is formed.

It should be kept in mind that when developing a plan, it is advisable to implement simultaneously no more than two business processes. The choice of implemented business processes should be based on an analysis of improvement priorities based on the strategic enterprise business objectives.

The implementation of the created ideal business process model includes:

- Step-by-step introduction of business processes "as it should be".

- In-time control of the implementation plan.

- Quality control of the created business processes.

- Correction of business process models on the practical experience basis.

- Changes in the organizational structure of the enterprise, the duties of performers.

- Development of new documentation (job descriptions created on the basis of business processes).

- Control of the successful implementation of new business processes.

Work on the implementation of the ideal business processes model is done strictly in accordance with the plan. The Contractor monitors the implementation of recommendations for the introduction and conformity of business processes to the models "as it should be". The quality control of the implementation of the business processes of the ideal model is accomplished by carrying out inspections of measurements of business process performance indicators. If necessary, the working group adjusts the developed business 
process models and associated documentation. It should be noted that for a certain period, old and new business processes running in parallel are allowed.

To create a continuous network of organization's business processes, an important management principle is implemented: every business process owner is responsible not only for the course and result of the process, but also for delivering the product to the consumer.

It is very important at this stage to create a set of job instructions, since the existing instructions on the vast majority of enterprises have a number of significant drawbacks:

- Absence of incoming and outgoing documents.

- No sequence of finished functions is documented.

- There is no description of initiating and trailing events.

- The results of each function are not documented.

- Areas of responsibility and employees' promotion for each function, etc. are not defined.

At this step, it is important to maintain detailed documentation of the work: business process analysis protocols, unit case files, quality assurance programs, vendor evaluation tables, internal audit protocols, variance analysis protocols, etc. All this documentation is developed for the specific organization by the working group. As a result of the work, a report containing recommendations on maintaining efficiency and quality control of the implemented business processes is generated and sent (Kukartsev \& Gorlevsky, 2014).

The analysis of actual improvements in business processes is carried out. If the changes do not produce the planned effect, the analysis of ideal models is repeated, after which the cycle for the introduction of improved business processes is repeated. If the models are implemented successfully, work on improving business processes is resumed and the algorithm is repeated again, because the process of improving the enterprise managed activity on the basis of a process approach is continuous.

The procedure of implementing improved business processes requires further elaboration and testing, since this is a large labour-consuming task that requires carrying out individual scientific research and practical developments. In the meantime, this step was only outlined in general terms, since without it the method of managing the RSI enterprise business processes would be incomplete.

\section{Conclusion}

The state of innovative and productive potential at the RSI enterprises, characterized by insufficient competitiveness in foreign markets, ineffective majority of science-intensive enterprises management and the insufficient level of planned budget investments for the development of the defence industry complex lead to the need to develop methods for managing innovative business processes of the RSI enterprises (Gorlevsky \& Kukartsev, 2014, pp. 194-198). This will allow one to take into account the nature and conditions for the implementation of innovative work, as well as ensure not only the effective use of limited resources, but also the sustainable development of the RSI enterprises and the industry as a whole, which will successfully solve the problems of enhancing the country's defence capability. 
Despite the fact that the methodology proposed in the article is based on well-known principles, some of which have already been successfully used in one form or another by industry enterprises, it is new since it allows the RSI enterprises to transfer to process management of innovative working activities. As a result, the enterprise can improve innovative business processes on an ongoing basis, which allows it to increase its competitiveness.

\section{References}

Baklanov, A.G. (2007) Market and marketing of aerospace products in conditions of instability, Moscow, KDU.

Batkovskiy, A. M., Makarov, Y. N., Semenova, E. G., Fomina, A. V., Khrustalev, E. I. (2015). Economic protection of secure operation and development of companies in the rocket and space industry, Mediterranean Journal of Social Sciences, 6(4), 414.

Becker, J., Vilkova, L., Taratukhina, V., Kugeler, M., Rosemann, M. (2007). Process Management, Moscow, Eksmo.

Boyko, A. A., Kukartsev, V. V., Tynchenko, V. S., Nasyrov, I. R., Kukartsev, V. A. (2018). State and trends of depreciation strategy of rocket and space industry enterprises formation, International Conference "Actual Issues of Mechanical Engineering" (AIME 2018), Advances in Engineering Research, 157, 607-611.

Chubai, V. M. (2010). Analysis of innovation potential of a machine-building enterprise in the process of formation and implementation of innovation strategy, Actual problems of economics, 110, 183-190.

Danilina, H., Mingaleva, Z. (2013). Improving of innovation potential efficiency of industrial enterprises, Middle East Journal of Scientific Research, 13, 191-194.

Gorlevskiya, K. I., Ogurchenok, I. V. (2014). Scientific approaches to management of business processes of the enterprise of the space-rocket industry, Current problems of aircraft and astronautics, 2(10), 113-114.

Gorlevsky, K. I., Kukartsev, A. V. (2014). Regulation of innovative business processes of the enterprise of the rocket and space industry, Bulletin of the Siberian State Aerospace University, 1(53), 194-198.

Kukartsev, A. V., Gorlevsky, K. I. (2014). Principles of management of innovative business processes of an enterprise in the rocket and space industry, Economics and management of control systems, 1(11), 44-52.

Meskon, M., Albert, M., Hedouri, F. (2009). Fundamentals of Management, Moscow, Williams.

Moller, C. (2006). Enterprise systems management and innovation: Impact on the research agenda, 8th International Conference on Enterprise Information Systems (ICEIS 2006), Proceedings DISI, 87 94.

Pabian, A., Pabian, B. (2014). Sustainable management of an enterprise - functional approach, Polish Journal of Management Studies, 10(1), 98-107.

Repin, V., Eliferov, V. (2013). Process approach to management. Business process modelling, Moscow, Mann, Ivanov and Ferber.

Tarasenko, M. V. (1996). Evolution of the Soviet space industry, Acta astronautica, 38(4-8), 667-673.

Yeremeev, D. V. (2013). Conceptual model of the relationship of competitiveness of high-tech enterprises and its innovative potential, Bulletin of Magnitogorsk State Technical University, 3, 101-106. 